\title{
Análise dos fatores atrelados ao atraso vacinal em crianças: um olhar à luz das evidências
}

Analysis of factors linked to vaccine delay in children: a look at the light of evidence

Análisis de factores vinculados al retraso de la vacuna en niños: una mirada a la luz de la evidencia

Ana Beatriz de Oliveira Vieira Matos ORCID: https://orcid.org/0000-0003-3604-661X Christus Faculdade do Piauí, Brasil E-mail: biabeatriz62@hotmail.com

Evaldo Sales Leal

ORCID: https://orcid.org/0000-0002-1424-9048

Christus Faculdade do Piauí, Brasil

E-mail: evaldosleal@hotmail.com

Bruna Daniella de Sousa de Lima ORCID: https://orcid.org/0000-0002-8321-1780

Universidade Estadual de Londrina, Brasil E-mail: enf.brunadaniella@uel.br

Maria Kallyne Carvalho Holanda ORCID: https://orcid.org/0000-0003-0797-2235

Christus Faculdade do Piauí, Brasil

E-mail: fisio.mariakallyne@gmail.com

Ana Pedrina Freitas Mascarenhas ORCID: https://orcid.org/0000-0003-4481-4551 Faculdade de Ciências da Saúde do Trairi, Brasil E-mail: anapedrinajp@ hotmail.com

Wedja Kalyandra Marques de Lima Ferreira ORCID: https://orcid.org/0000-0001-5403-0959 Centro Universitário Maurício de Nassau, Brasil E-mail: wedjabiju@hotmail.com

Shimeny Lima Lucena Dantas ORCID: https://orcid.org/0000-0002-8652-9532 Universidade Estadual da Paraíba, Brasil E-mail: shimenylima@hotmail.com

Ana Carla da Silva Linhares ORCID: https://orcid.org/0000-0001-9435-8916 Faculdade Santa Maria, Brasil E-mail: carlinhatds@hotmail.com

Elisane Alves do Nascimento ORCID: https://orcid.org/0000-0002-6318-1772 Faculdade Uninassau, Brasil

E-mail: elisaneanascimento@gmail.com

Glenda Suellen Matos Cruz ORCID: https://orcid.org/0000-0003-1933-1937 Faculdade Dom Pedro II, Brasil E-mail: gl.suellen@ hotmail.com

Luiz Carlos Pereira de Sousa ORCID: https://orcid.org/0000-0002-5742-6315 Centro Universitário de Patos, Brasil E-mail: luizcarlosperreira.15@gmail.com

Gabriel Siqueira Matheus ORCID: https://orcid.org/0000-0002-1373-9221 Universidade Estadual de Maringá, Brasil

E-mail: gabrielsm1@live.com

Karine Lustosa Araújo ORCID: https://orcid.org/0000-0002-0779-0119 Christus Faculdade do Piauí, Brasi

E-mail: karinelustosa855@gmail.com

Klessiane Mendes de Fontes ORCID: https://orcid.org/0000-0002-5058-9613 Hospital Universitário Lauro Wanderley, Brasil

E-mail: klessianemendes@gmail.com 
Clara Janyelle Gomes de Carvalho ORCID: https://orcid.org/0000-0001-5320-8647 Faculdade de Ciências da Saúde do Trairi, Brasil E-mail: claracarvalhofisio@gmail.com

\begin{abstract}
Resumo
É indubitável a relevância que as vacinas têm na proteção à saúde e na prevenção de doenças imunopreveníveis, sobretudo durante a infância. Objetivou-se discutir, à luz das evidências, quais os fatores atrelados ao atraso vacinal em crianças. O presente estudo tratou-se de uma revisão integrativa, a coleta de dados ocorreu pela busca da melhor evidência dentro da literatura existente. A busca foi realizada mediante a pesquisa nas seguintes bases de dados: MEDLINE®, LILACS e BDENF. Os critérios de inclusão utilizados foram artigos científicos, dissertação de mestrado e teses de doutorado, estudo nos idiomas português, inglês e espanhol, texto completo disponível eletronicamente e de forma gratuita, publicados nos últimos 5 anos. Foram excluídos os trabalhos que não atendiam à questão norteadora de pesquisa. Foram selecionados 07 artigos para análise da revisão integrativa, sendo que para melhor discussão foram divididos em três categorias: fatores relacionados aos aspectos sociodemográficos, fatores relacionados aos aspectos estruturais das UBS e fatores relacionados à atuação da ESF e à saúde das crianças que contribuem para o atraso vacinal. Os autores revelam também que a redução da cobertura vacinal se tem por causas multifatoriais. Compreende-se que, apesar dos importantes achados desse estudo que subsidiarão a gestão e políticas voltadas à cobertura vacinal, há algumas limitações que não viabilizam o estudo. Diante do exposto, evidenciando a relevância e o impacto que a imunização exerce sobre a saúde, é indispensável que informações concisas e atuais sobre imunização sejam compartilhadas, assim como o desenvolvimento de estudos que possam colaborar com melhorias e a elucidação de outros desafios relacionados às ações de imunização.
\end{abstract}

Palavras-chave: Cobertura vacinal; Criança; Pesquisa sobre serviços de saúde.

\begin{abstract}
The relevance that vaccines have protection against health and prevention of vaccine-preventable diseases is undoubtedly, especially during childhood. The objective was to discuss, in the light of detection, what are the factors linked to vaccination delay in children. The present study deals with an integrative review, a collection of data that took place in search of the best evidence within the existing literature. A search was performed using a search in the following databases: MEDLINE®, LILACS and BDENF. The inclusion requests used were scientific articles, master's and doctoral dissertations, studies in Portuguese, English and Spanish, full text available electronically and free of charge, published in the last 5 years. Works that do not meet the guiding research question were excluded. Seven articles were selected for analysis of the integrative review, and for the best discussion they were divided into three categories: factors related to sociodemographic aspects, factors related to aspects of UBS statistics and factors related to the performance of the ESF and the health of children who contributed vaccination delay. The authors also reveal that the reduction in vaccination coverage is due to multifactorial causes. Understand that, despite the important findings of this study that subsidizes the management and policies aimed at vaccination coverage, there are some configurations that do not enable the study. Given the above, the evidence and the impact that immunization has on health, it is essential that information and statistics on immunization be shared, such as the development of studies that can collaborate with improvements and the elucidation of other issues related to immunization actions.
\end{abstract}

Keywords: Vaccine coverage; Kid; Health services research.

\begin{abstract}
o
La importancia de las vacunas para proteger la salud y prevenir enfermedades prevenibles por vacunación, especialmente durante la infancia, es incuestionable. El objetivo fue discutir, a la luz de la evidencia, qué factores están relacionados con el retraso de la vacunación en los niños. El presente estudio fue una revisión integradora, la recolección de datos ocurrió a través de la búsqueda de la mejor evidencia dentro de la literatura existente. La búsqueda se realizó mediante búsquedas en las siguientes bases de datos: MEDLINE®, LILACS y BDENF. Los criterios de inclusión utilizados fueron artículos científicos, tesis de maestría y tesis doctorales, estudio en portugués, inglés y español, texto completo disponible de forma electrónica y gratuita, publicados en los últimos 5 años. Se excluyeron los trabajos que no cumplieron con la pregunta orientadora de la investigación. Se seleccionaron siete artículos para el análisis de la revisión integradora, y para una mejor discusión se dividieron en tres categorías: factores relacionados con aspectos sociodemográficos, factores relacionados con aspectos estructurales de la UBS y factores relacionados con el desempeño de la ESF y la salud de niños que contribuyen al retraso de la vacuna. Los autores también revelan que la reducción de la cobertura de vacunación se debe a causas multifactoriales. Se entiende que, a pesar de los importantes hallazgos de este estudio que respaldarán la gestión y las políticas orientadas a la cobertura de vacunación, existen algunas limitaciones que no hacen factible el estudio. A la luz de lo anterior, evidenciando la relevancia e impacto que tiene la inmunización en la salud, es fundamental que se comparta información concisa y actualizada sobre inmunización, así como el desarrollo de estudios que puedan colaborar con mejoras y el esclarecimiento de otros desafíos relacionados con la inmunización. las acciones de la inmunización.
\end{abstract}

Palabras clave: Cobertura de la vacuna; Niño; Encuesta de servicios de salud. 


\section{Introdução}

Nos últimos anos, entre as maiores ascensões analisadas na área da saúde, a imunização vem se apoderando de um âmbito gradativamente maior. $\mathrm{O}$ avanço nas pesquisas científicas na área da saúde tem se somado aos estudos epidemiológicos e sociológicos, os quais evidenciam o grande impacto que as vacinas têm representado para a sociedade, demonstrando um dos principais elementos de promoção de saúde e prevenção de doenças (Feijó et al., 2006).

É indubitável a relevância que as vacinas têm na proteção à saúde e na prevenção de doenças imunopreveníveis, sobretudo durante a infância. Em resultância, as autoridades de saúde, de todo o mundo, estabeleceram calendários específicos de vacinas de acordo com cada faixa etária. No Brasil, o Ministério da Saúde implementa programas de imunização e viabiliza, periodicamente, campanhas com a finalidade de controlar e erradicar doenças a partir da vacinação maciça (Silveira et al., 2007).

Desde o começo do século XX, as vacinas, juntamente com diversas intervenções de vigilância, vêm sendo encarregadas pela extinção e monitoramento de enfermidades imunopreveníveis, a exemplo da erradicação da varíola e da descontinuação da transmissão da poliomielite. Em 1973, foi instituído o Programa Nacional de Imunizações (PNI), atribui-se como sendo o principal mecanismo de viabilização dos resultados alcançados, torna-se efetivo por intermédio de atividades coordenadas de planejamento, qualificação, infraestrutura e logística, comprometidas com a entrega à população de um produto de excelência, com todas as suas características e especificidades preservadas (Brasil, 2015).

A imunização continua sendo uma das intervenções de saúde mais impactante e econômica. O PNI teve como objetivo, uniformizar, implantar, inspecionar e avaliar as ações de imunização, além de propor políticas e estratégias para viabilizar coberturas vacinais adequadas em todo o país. Incube ainda ao Programa, a aquisição, condicionamento, distribuição e aplicação de imunobiológicos contemplados no calendário básico de vacinação ou indicados em situações especiais, além da vigilância de eventos adversos e garantia da segurança dos procedimentos operacionais (Brasil, 2003).

A saúde pública, com funções nas três esferas (federal, estadual e municipal), construiu a base de um sistema de imunização que garante que políticas e programas de imunização promovam acesso equitativo a vacinas seguras e eficazes (Comitê Consultivo Nacional Para Vacinas, 2013). O PNI, é um programa com múltiplos avanços, como por exemplo, a inclusão de novas vacinas e de grupos alvos, assim como a sua modernização no campo da informática e da informação (Texeira; Rocha, 2010).

A vacina é uma substância preparada com bactérias ou vírus (ou fragmentos dele) mortos ou enfraquecidos. Ao adentrar o corpo do ser humano, a vacina produz uma reação (imunização) do sistema imunológico, proporcionando a formação de anticorpos (leucócitos) contra aquela substância (Lemos, 2011). Desta forma, a vacina é um artifício preventivo de extrema relevância a todos os habitantes do mundo, que proporciona além da proteção individual contra sérias enfermidades, a proteção à comunidade, restringindo a disseminação de agentes infecciosos. A vacina para crianças e idosos é indispensável, em virtude que estes indivíduos se encontram, do ponto de vista imunológico, mas susceptíveis às doenças (Oliveira et al., 2010).

A população brasileira tem alcance integralmente a todas as vacinas preconizadas pela Organização Mundial de Saúde (OMS). A partir da criação do PNI, o programa procura a inserção social, contemplando a toda a população, sem distinção de qualquer natureza. Atualmente, o Brasil desfruta de mais de 36 mil salas de vacinação difundidas por todo território, sendo administrado anualmente 300 milhões de imunobiológicos, entre eles, é disponibilizado 27 vacinas, 13 soros heterólogos (imunoglobulinas animais) e 4 soros homólogos (imunoglobulinas humanas), todos ofertados de graça com materiais confiáveis e de qualidade (Rocha, 2015).

A produtividade do programa é averiguada através da cobertura vacinal (CV) de uma amostra de indivíduos, está instruído pelo sistema de saúde, pelo próprio programa de imunização e pelas particularidades da população. A CV tem sido estimada no país com base nas informações catalogadas pelas Unidades Básicas de Saúde (UBS), estando sujeita à importante 
erro de registro, transcrição, estimativa de população alvo e outros. Episódios de epidemias em vigor de estimativas de coberturas altas, bem como os inquéritos domiciliares executados, evidencia a inconsistências dessas estimativas (Brasil, 2007).

A CV não atinge nenhuma meta no calendário infantil desde de 2018, as últimas metas de imunização para o público infantil atingidas no país, em 2018, foram de 99,72\% do público-alvo para a BCG, e de 91,33\% para o da vacina contra o Rotavírus humano. Para ambas, a meta é superar os 90\%, índice que não foi atingido em 2019, apesar de terem continuado acima dos $80 \%$. Até outubro de 2020, a taxa de imunização da BCG chegou a 63,88\%, e a vacina contra o Rotavírus, a 68,46\%. A maior CV atingida no calendário infantil no mesmo período foi a vacina Pneumocócica, com 71,98\%. Em 2019, a mesma vacina chegou a 88,59\% do público-alvo. Entre as vacinas disponibilizadas no calendário infantil, que inclui a segunda dose da Tríplice viral, metade não atinge as metas desde 2015, o que inclui a vacina contra poliomielite (Lisboa, 2020).

Dentre os fatores associados para a não vacinação ocasionando assim, a redução da cobertura vacinal salienta-se: extremidades de idade materna, maior número de filhos, baixo grau de instrução materna, habitação há menos de 1 ano na área, maior número de moradores no aposento, domicílio em área rural, baixa renda, ausência de informação acerca das doenças preveníveis por imunização, impedimento de deslocamento, embates laborais ocasionados pela perda de dias de expediente para o cuidado dos filhos, inexistência de seguro-saúde e existência de doença na criança (Mota, 2016).

Em 2020, de acordo com os novos dados da OMS e da UNICEF, mais de 23 milhões de crianças perderam vacinas que salvam vidas, 3,7 milhões a mais do que em 2019. Dados abrangentes que refletem a interrupção do serviço global devido à COVID-19, de forma preocupante, a maioria dessas crianças - até 17 milhões - provavelmente não recebeu nenhuma vacina durante o ano, ampliando as já imensas inequidades no acesso às vacinas (Opas, 2020). Diante das considerações até aqui elencadas e de todo o contexto envolvido na elucidação destes delineamentos questiona-se quais os fatores atrelados ao atraso vacinal em crianças? Portanto, o objetivo deste estudo consiste em discutir, à luz das evidências, quais os fatores atrelados ao atraso vacinal em crianças.

Acredita-se que é essencial identificar os principais fatores que ocasionam a negligência para a realização desta ação de importância ímpar para as crianças, considerando-se que exista um declínio na CV no país. A escolha da temática se deu pela afinidade pelo tema, e pela área, bem como pela inquietação sobre a redução da CV. Percebe-se a necessidade de maiores embates no campo conceitual e subjetivo dos processos avaliativos dos serviços de saúde, sendo assim, avaliar os fatores atrelados a CV, colaborará para aperfeiçoamentos nas ações e nos serviços, respaldando políticas públicas em saúde voltadas para o enfrentamento do objeto de estudo. Esta pesquisa é de grande relevância para a sociedade como também para gestores, profissionais e público leigo, pois por meio dele será mostrada a situação da imunização de crianças do município.

A identificação dos fatores que influenciam a cobertura vacinal poderá contribuir para a redução de gastos atrelados aos inúmeros agravos advindos da não imunização adequada de crianças. Assim, uma vez que se pode reduzir a carga de morbimortalidade por doenças imunoevitáveis, como por exemplo, de doenças até então controladas e/ou erradicadas em tempos mais remotos e que podem retornar ao cenário atual.

\section{Metodologia}

O presente estudo tratou-se de uma revisão integrativa, a qual é um método de revisão mais amplo, pois permite incluir literatura teórica e empírica bem como estudos com diferentes abordagens metodológicas (quantitativa e qualitativa) (Pompeo et al., 2009). A coleta de dados ocorreu pela busca da melhor evidência dentro da literatura existente, que inclui a pesquisa de artigos originais em periódicos e dentro das bases de dados confiáveis, com o objetivo de encontrar referencias que condizem com o tema abordado mediante a formulação do problema (Pompeo et al., 2009; De-La-Torre-Ugarte-Guanilo et al., 2011).

A busca foi realizada mediante a pesquisa nas seguintes bases de dados: Sistema Online de Busca e Análise de Literatura Médica (Medical Literature Analysis and Retrieval System Online) (MEDLINE®), Literatura Latino-Americana e do Caribe em 
Ciências da Saúde (LILACS), Base de dados em Enfermagem (BDENF). As palavras-chaves utilizadas seguiram a descrição dos termos Descritores em Ciência em Saúde (DeCS) e Medical Subject Heading (MESH) nos idiomas inglês e português, sendo estes: cobertura vacinal, criança e pesquisa sobre serviços de saúde. A fim de se realizar a busca integrada utilizou-se o conectivo "and" unindo os descritores. Nessa etapa não houve utilização de outros filtros no intuito de abordar toda a literatura disponível dentro do foco de interesse. As duplicatas foram resolvidas após verificação de todos os títulos dos trabalhos.

Os critérios de inclusão utilizados foram artigos científicos, dissertação de mestrado e teses de doutorado, estudo nos idiomas português, inglês e espanhol, texto completo disponível eletronicamente e de forma gratuita, ser estudo do tipo original, de revisão bibliográfica, estudo de caso, relato de experiência, descritivo observacional e randomizado, estudos que abordavam a temática proposta. Pela particularidade do tema e pelo número reduzidos de artigos na literatura sobre o tema, foram incluídos trabalhos científicos publicados no período de (2015 a 2020) disponibilizados de forma integral, com livre acesso ao texto e que estivessem adequados ao tema proposto. Foram excluídos os trabalhos que não atendiam à questão norteadora de pesquisa, estudo em duplicatas em mais de uma base de dados, pesquisas em animais e estudos in vitro.

Após a realização da busca nas bases de dados MEDLINE, LILACS, e BDENF, utilizando as estratégias de busca anteriormente mencionadas, chegou-se a um total de 02 artigos na MEDLINE, 20 artigos na LILACS, e 03 artigos na BDENF. Ao aplicar os critérios de inclusão e exclusão, chegou-se a um total de 01 artigos na MEDLINE, 05 artigos na LILACS, e 01 artigos na BDENF, como detalhado no fluxograma abaixo. Os dados foram organizados em quadro síntese para posterior discussão, sendo que as informações contidas dividiram-se da seguinte forma: título, autores, revista, ano e os principais achados. 
Fluxograma - Distribuição da estratégia de busca dos achados nas bases de dados.

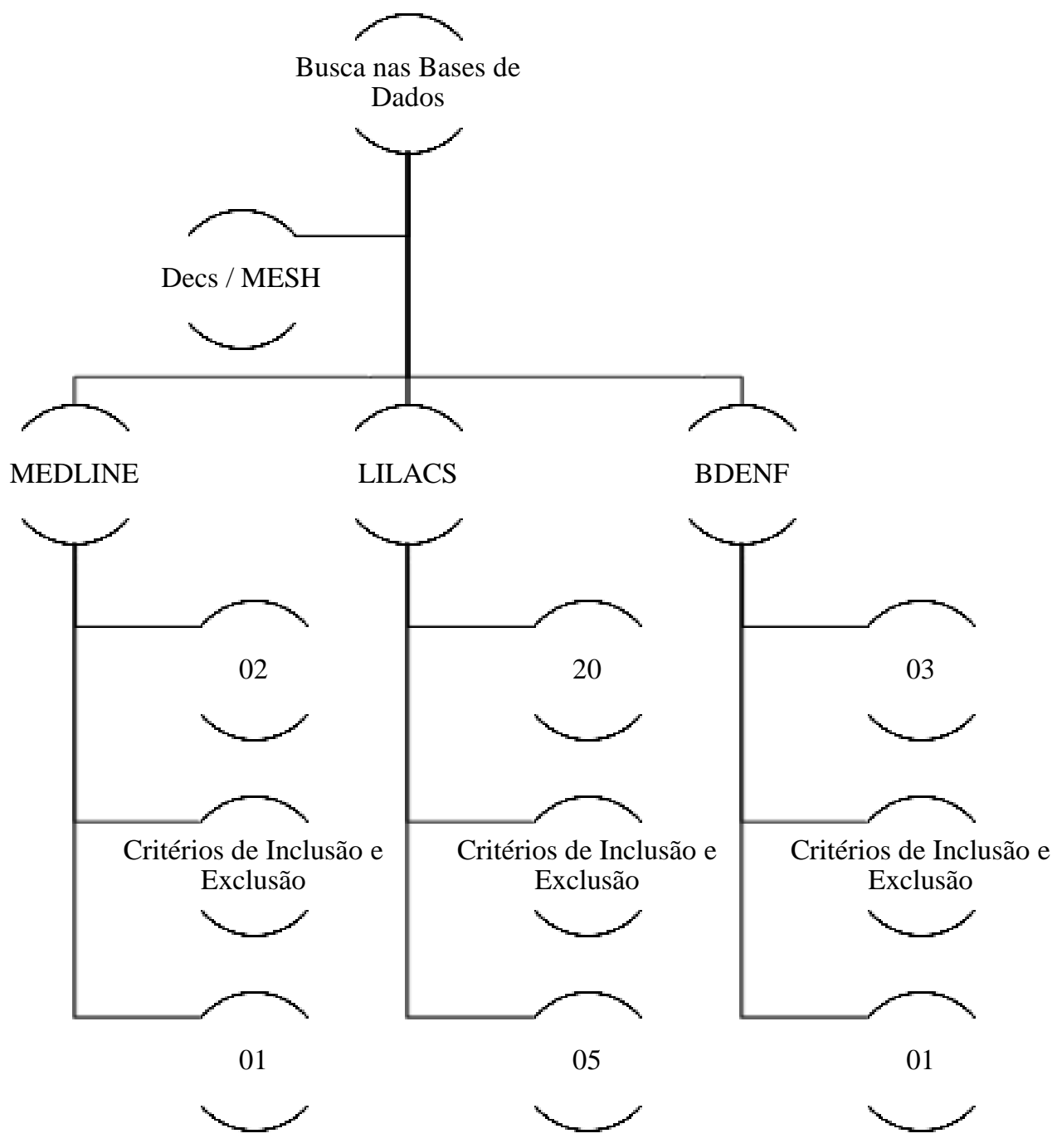

Fonte: Autores (2021).

Vale ressaltar que todas as normas de autoria foram respeitadas, referenciando os autores citados no estudo, conforme normas de Associação Brasileira de Normas Técnicas (ABNT).

\section{Resultados e Discussão}

Inicialmente foi construído um quadro para expor os estudos contendo informações como ano, título, autores e local de publicação. Após as pesquisas nas bases de dados por meio dos descritores de inclusão e exclusão estabelecidos, foram selecionados 07 artigos presentes no (Quadro 1) para análise da revisão integrativa. 
Quadro 1- Artigos para análise da Revisão Integrativa.

\begin{tabular}{|c|c|c|c|c|}
\hline Estudo & Ano & Título & Autores & Local De Publicação \\
\hline 01 & 2019 & $\begin{array}{l}\text { Imunização Contra Papilomavirus } \\
\text { Humano Em Escolas Municipais }\end{array}$ & Feitosa L.G. et al. & Revista De Enfermagem UFPE On Line \\
\hline 02 & 2018 & $\begin{array}{l}\text { Avaliação De Coberturas Vacinais De } \\
\text { Crianças Em Uma Cidade De Médio } \\
\text { Porte (Brasil) Utilizando Registro } \\
\text { Informatizado De Imunização }\end{array}$ & $\begin{array}{l}\text { Ferreira V. L. R. et } \\
\text { al. }\end{array}$ & Cadernos De Saúde Pública (On Line) \\
\hline 03 & 2018 & $\begin{array}{l}\text { Qual A Importância Da Hesitação } \\
\text { Vacinal Na Queda Das Coberturas } \\
\text { Vacinais No Brasil? }\end{array}$ & Sato A.P.S. & Revista de Saúde Pública \\
\hline 04 & 2017 & $\begin{array}{l}\text { Coberturas Vacinais Por } r \text { Doses } \\
\text { Recebidas E Oportunas Com Base Em } \\
\text { Um Registro Informatizado De } \\
\text { Imunização, Araraquara-Sp, Brasil, } \\
2012-2014\end{array}$ & Tauil M.C. et al. & Epidemiologia e Serviços de Saúde \\
\hline 05 & 2017 & $\begin{array}{l}\text { Retraso Del Esquema Vacunal En Niños } \\
\text { Menores De } 5 \text { Años En Zona Marginal. }\end{array}$ & $\begin{array}{l}\text { Dominguez G.C.S. } \\
\text { et al. }\end{array}$ & Revista Del Nacional (Itauguá) \\
\hline 06 & 2015 & $\begin{array}{l}\text { Análise Da Situação Vacinal De Crianças } \\
\text { Pré-Escolares Em Teresina (Pi) }\end{array}$ & $\begin{array}{l}\text { Fernandes, A.C.N et } \\
\text { al. }\end{array}$ & Revista Brasileira de Epidemiologia \\
\hline 07 & 2015 & $\begin{array}{l}\text { Avaliação Da Cobertura Vacinal Em } \\
\text { Crianças De } 2 \text { Meses A } 5 \text { Anos Na } \\
\text { Estratégia Saúde Da Família }\end{array}$ & $\begin{array}{l}\text { Cardoso M.D.T. et } \\
\text { al. }\end{array}$ & Revista APS \\
\hline
\end{tabular}

Fonte: Autores (2021).

Pode-se observar pelos dados do Quadro 1, a escassez de estudos recentes do tema proposto, principalmente na literatura inglesa e espanhola. Todos os artigos encontrados estão representados no quadro acima com predomínio da língua portuguesa (5), sendo apenas 1 artigo na língua inglesa e 1 na língua espanhola. Observou-se também, que 1 (14,3\%) dos artigos foram publicados no ano de 2019, de 6 (85,7\%) artigos datados entre os anos de 2015 a 2018.

Com os dados obtidos através da análise dos artigos, observou-se que de 2015 a 2019 o menor número de publicações concentrou-se no ano de 2019. Em relação à natureza dos artigos, 1 era estudo descritivo do tipo de relato de experiência, 2 estudos descritivos, 1 tese, 1 estudo observacional, descritivo de corte transversal, 1 estudo seccional e 1 estudo transversal e descritivo.

Os autores destes trabalhos apontaram a baixa cobertura vacinal pode ser de causa multifatorial, no qual serão detalhados posteriormente. No Quadro 2, pode-se observar os principais achados dos estudos selecionados para a presente pesquisa.

Quadro 2 - Caracterização dos principais achados dos estudos incluídos na revisão.

\begin{tabular}{|l|l|}
\hline Estudos & Principais Achados \\
\hline 01 & $\begin{array}{l}\text { Conhecimento insatisfatório sobre os imunobiológicos pela população assistida; } \\
\text { Falta de adesão quando solicitados à população para análise do cartão vacinal; } \\
\text { Suma importância a criação e fortalecimento das parceiras entre a saúde (equipes de saúde) e educação (escolas); } \\
\text { Facilitação do acesso ao serviço de saúde, por meio de estratégias de busca ativa e descida nas escolas. }\end{array}$ \\
\hline 02 & $\begin{array}{l}\text { Influência nas coberturas vacinais sempre que se muda o calendário de imunização; } \\
\text { Introdução de novas vacinas no calendário de imunização; } \\
\text { Utilização da rede privada para efetuar a vacinação; }\end{array}$ \\
\hline
\end{tabular}




\begin{tabular}{|c|c|}
\hline & lta de acompanhamento por meio de sistema de informação mais amplo e consistente. \\
\hline 03 & $\begin{array}{l}\text { Hesitação vacinal como algo que está em crescente no contexto brasileiro, uma vez que já se observa este movimento ao } \\
\text { redor do mundo; } \\
\text { Utilização de recusa às vacinas atreladas ao risco que elas podem trazer baseada em evidências empíricas; } \\
\text { Os movimentos anti-vacinas que vem crescendo principalmente em países de alta renda; } \\
\text { Impactos negativos serão observados principalmente em países de média e baixa renda. }\end{array}$ \\
\hline 04 & $\begin{array}{l}\text { Necessidade de um sistema de monitoramento oportuno de vacinação em idade apropriada; } \\
\text { Disponibilidade de imunobiológicos no momento da procura pela imunização no serviço de saúde; } \\
\text { Mudanças de esquema por períodos, por falta de imunobiológicos da rotina usual; } \\
\text { Recomendação para a criança receber doses de imunobiológicos especiais; } \\
\text { Vacinas oferecidas pelo setor privado diferentes daquelas oferecidas pelo setor público; }\end{array}$ \\
\hline 05 & $\begin{array}{l}\text { É essencial questionar o status vacinal em todos os níveis de atenção, tanto na atenção básica quanto nos pacientes } \\
\text { hospitalizados, naqueles que atendem os serviços de emergência ou uma consulta especializada, a fim de detectar a } \\
\text { população vulnerável ao atraso na vacinação; } \\
\text { O motivo predominante do atraso foram as doenças diagnosticadas pelos médicos, as falsas contra-indicações e a perda } \\
\text { do cartão; } \\
\text { Os fatores envolvidos no cumprimento do esquema de vacinação em crianças menores de um ano foram renda } \\
\text { econômica, despesas de viagem para vacinar a criança, crença em vacinas, desconhecimento das vacinas gratuitas, } \\
\text { tratamento da equipe de enfermagem, informações sobre consultas subsequentes e reações pós-vacinais; } \\
\text { Outros fatores, como ser mãe adolescente, aliada a um baixo nível de escolaridade e pertencer a um grupo étnico indígena, } \\
\text { influenciam diretamente a taxa de abandono do esquema de vacinação para crianças menores de um ano de idade. }\end{array}$ \\
\hline 06 & $\begin{array}{l}\text { Os atrasos vacinais podem desencadear problemas sérios em bem pouco tempo, se passarem despercebidos; } \\
\text { A comunidade precisa muito mais de apoio, conforto e atenção em suas dificuldades que de procedimentos e atividades } \\
\text { bem executadas; } \\
\text { Quanto aos dados sociodemográficos, a renda per capita não revelou associação em relação ao atraso vacinal; } \\
\text { As pessoas que frequentam a rede privada de assistência à saúde, com maior grau de escolaridade, têm chances } \\
\text { consideráveis de não-vacinação e recusa em vacinar suas crianças; } \\
\text { Dentre os motivos expressados pelos pais para o atraso vacinal e/ou para a não-vacinação, os mais frequentes foram falta } \\
\text { de vacinas e negligência. } \\
\text { Observou-se que quanto mais doses a vacina tiver no esquema, maiores são as chances de atrasos vacinais } \\
\text { Muitas equipes operam com deficiências, tanto estruturais quanto aquelas ligadas às atividades desenvolvidas pelos } \\
\text { profissionais; } \\
\text { Tanto profissionais quanto pais/responsáveis focam o cuidado em situações de agravo à saúde da criança, negligenciando } \\
\text { a atenção primária e o acompanhamento periódico; } \\
\text { Os profissionais atribuem esse fator à sobrecarga de trabalho, ao despreparo e à escassez de recursos, a fim de justificar } \\
\text { falhas na verificação das cadernetas das crianças durante as consultas; } \\
\text { Pais/cuidadores que não levam seus filhos às consultas rotineiras nas UBS ou levam apenas eventualmente são fortes } \\
\text { candidatos a descuidar de sua vacinação. }\end{array}$ \\
\hline 07 & $\begin{array}{l}\text { Percebe-se que o atraso vacinal realmente se relaciona com os fatores de riscos considerados, tais como baixa renda } \\
\text { familiar, maior número de filhos, baixa escolaridade materna e presença de doença na criança, entre outros; } \\
\text { Notou-se que a menor estrutura da UBSF e menor equipe de saúde também contribuem para a falha do esquema vacinal; } \\
\text { Para se obter maior êxito na cobertura vacinal, cabe aos profissionais de saúde uma participação mais ativa na busca de } \\
\text { crianças em falta com a vacinação, por meio da revisão sistemática dos cartões, de uma maior efetividade nas visitas } \\
\text { domiciliares, cabendo um maior comprometimento aos agentes de saúde; } \\
\text { Outra estratégia para o aumento da cobertura vacinal é a melhoria das atividades de educação em saúde, com o intuito } \\
\text { de difundir informações aos grupos-alvo, uma vez que a falta de conhecimento e as crenças da população contribuem } \\
\text { para o atraso vacinal. }\end{array}$ \\
\hline
\end{tabular}

Fonte: Autores (2021). 


\subsection{Categorias de análises dos principais achados dos estudos incluídos na revisão}

\subsubsection{Fatores relacionados aos aspectos sociodemográficos associados ao atraso vacinal}

O estudo 1, aponta que a recusa vacinal de alguns imunobiológicos estão relacionados à falta de conhecimento sobre a enfermidade, as formas de transmissão e possíveis complicações, desconhecimento sobre os benefícios da vacina e a preocupação dos responsáveis com os efeitos adversos. A impossibilidade de ir até a unidade de saúde para vacinar-se em conjunto com os fatores expostos acima podem acarretar a baixa cobertura vacinal (Feitosa et al., 2019).

O estudo 3, ressalta características contextuais e individuais que influenciam diretamente na situação vacinal. O estudo evidencia as influências contextuais incluindo os aspectos históricos, geográficos, políticos, socioeconômicos, culturais, religiosos e de gênero, assim como a comunicação e mídia, influência de líderes e a percepção sobre a indústria farmacêutica (Sato, 2018).

As influências individuais estão relacionadas a experiências prévias com vacinação, crenças e atitudes sobre saúde, confiança ao sistema de saúde, vínculo com profissionais de saúde, percepção de risco da vacina e visão da imunização como norma social contra a de que a vacinação não é necessária ou é nociva. O estudo enfatiza que a hesitação vacinal e seus determinantes variam ao longo do tempo e são específicos de cada contexto (Sato, 2018).

O estudo 5, enfatiza a violação do esquema de vacinação que aumentou consideravelmente, constituindo um grave problema de saúde pública. A aplicação oportuna de vacinas protege a população, o mais cedo possível, do contágio com agentes potencialmente letais, causando pelo menos grande morbidade. Ter proteção adequada é particularmente importante em doenças de circulação contínua, como o coqueluche e aquelas que provavelmente causam grandes surtos, como o sarampo (Dominguez et al., 2017).

O risco de adoecer devido a um atraso na vacinação depende do tipo de vacina, grau de transmissibilidade, circulação e gravidade da doença, além da presença de casos importados. Os fatores envolvidos no cumprimento do esquema de vacinação em crianças menores de um ano foram renda econômica, despesas de viagem para vacinar a criança, crença em vacinas, desconhecimento das vacinas gratuitas, tratamento da equipe de enfermagem, informações sobre consultas subsequentes e reações pós-vacinais (Dominguez et al., 2017).

Outros fatores apontados no estudo foram, ser mãe adolescente, aliada a um baixo nível de escolaridade e pertencer a um grupo étnico indígena, influenciam diretamente a taxa de abandono do esquema de vacinação para crianças menores de um ano de idade. Os grupos populacionais mais desfavorecidos são aqueles que tendem a ter menor cobertura vacinal, consultam com menos frequência e são o grupo com maior impacto nas oportunidades perdidas de vacinação (Dominguez et al., 2017).

O estudo 7, evidenciou-se que o atraso vacinal realmente se relaciona com os fatores de risco considerados, tais como baixa renda familiar, maior número de filhos, baixa escolaridade materna e presença de doença na criança, entre outros. Mas em contrapartida, destacou-se que o fator de risco relacionado à idade materna, o maior percentual de atraso foi observado na faixa etária entre 26 e 30 anos, contrariando os outros estudos citados na pesquisa (Cardoso et al., 2015).

\subsubsection{Fatores relacionados aos aspectos estruturais das UBS associados ao atraso vacinal}

No estudo 2 enfatiza-se que a introdução de novas vacinas no calendário de imunização foi observada a diminuição inicial das coberturas vacinais. No entanto, verificou-se elevação dessas em períodos de manutenção de um mesmo calendário vacinal. O estudo aponta que o aumento da cobertura vacinal observado ao longo dos anos pode está relacionado ao uso do Registro Informatizado de Imunização (RII) (Ferreira et al., 2018).

Os autores evidenciaram que o RII passou por constante melhoria e conta com ferramenta como avisos sobre validade de doses, diminuição de doses inválidas, relatório de faltosos/atrasados, permite a busca ativa para atualização de esquemas vacinais e relatórios de crianças com vacinas agendadas, aumentando a adesão e a oportunidade de vacinação. Além disso, a 
rapidez e o baixo custo são diferenciais, além da possibilidade de análise de validade das doses e oportunidades de vacinação (Ferreira et al., 2018).

O estudo 3 salienta que muitos fatores estão relacionados à queda da cobertura vacinal, seja o enfraquecimento do Sistema Único de Saúde ou aspectos técnicos como a implantação do novo sistema de informação de imunização. Entretanto, desde a década de 1990, as coberturas vacinais brasileiras são elevadas, o que reflete a boa aceitação por parte da população (Sato, 2018).

A princípio, contribuíram para essa conquista o aprimoramento do PNI e o avanço da pesquisa, desenvolvimento e produção de imunobiológicos no país. No entanto, esse cenário vem mudando rapidamente, onde é recorrente a reincidência de enfermidades anteriormente erradicadas, como por exemplo, epidemias de sarampo no ano de 2018, sendo confirmados mais de 1.500 casos nos estados de Roraima e Amazonas. Essa estatística é um alerta sobre o impacto da queda das coberturas vacinais (Sato, 2018).

O estudo 4 evidencia a utilização do RII, destacando-se a empregabilidade do instrumento para o alcance e manutenção de elevadas coberturas vacinais. Constatou-se que as coberturas vacinais ficaram acima de $90 \%$ para a maioria das vacinas analisadas no estudo. Contudo, foram observados percentuais mais baixos em relação às doses oportunas. As análises ao longo do tempo revelaram o atraso vacinal. O preenchimento de todas as doses de vacinas no RII pode ter levado a uma subestimação das coberturas vacinais. No entanto, ter considerado diferentes esquemas vacinais para o cálculo das coberturas e oportunidades vacinais, colaborou para a melhoria na qualidade de dados (Tauil et al., 2017).

A alta cobertura vacinal registrada no RII e a baixa duplicidade de registros reforçam sua utilidade para o monitoramento e controle das vacinas realizadas. Embora o RII tenha-se mostrado útil, a disponibilidade de imunobiológicos no momento da procura pela imunização no serviço de saúde, as mudanças de esquemas por período, decorrente da falta de imunobiológicos da rotina usual e até mesmo a oferta de vacinas diferentes no setor privado diferentes daquelas oferecidas pelo setor público, mostra a complexidade de se analisar os dados de cobertura (Tauil et al., 2017).

O estudo 6 destacou dentre os motivos expressados pelos pais para o atraso vacinal e/ou para a não vacinação, os mais frequentes foram faltas de vacinas e negligência. As perdas vacinais são possivelmente decorrentes do vencimento dos imunobiológicos, quebra de frascos e problema na Rede de Frio. De acordo com estudos, observou-se que quanto mais doses a vacina tiver no esquema, maiores são as chances de atrasos vacinais (Fernandes, A.C.N et al., 2015). No estudo 7, corroborando com os demais estudos, notou-se que a menor estrutura da UBSF e menor equipe de saúde também contribuem para a falha do esquema vacinal (Cardoso et al., 2015).

\subsubsection{Fatores relacionados à atuação da ESF e à saúde das crianças que contribuem para o atraso vacinal}

O estudo 1 apresentou o despreparo dos profissionais de saúde frente à atuação no ambiente escolar, sendo necessário a capacitação dos mesmos para assim, realizar práticas educativas para escolares e seus responsáveis, no intuito de elucidar a importância da vacinação, buscando minimizar o estigma da mesma e proporcionar o aumento da confiança para maior adesão à imunização. O esclarecimento de dúvidas e anseios minimiza a rejeição e aumenta a cobertura vacinal (Feitosa et al., 2019).

Sendo de suma importância a criação e fortalecimento das parceiras entre a saúde (equipes de saúde) e educação (escolas), pois isso oportuniza o acesso ao serviço de saúde, por meio de estratégias de busca ativa e descida nas escolas (Feitosa et al., 2019). O estudo 3 ressalta sobre o papel do profissional de saúde, corroborando com o estudo 1, que salienta intervenções educativas, evidenciando hesitação vacinal entre profissionais de saúde brasileiro. Do mesmo modo deve ser reforçada a seriedade da imunização na própria formação e educação continuada desses profissionais (Sato, 2018). 
O estudo 5 aponta que o motivo predominante de atraso vacinal foram enfermidades diagnosticadas por médicos e as falsas contraindicações, o que evidencia o despreparo por parte da equipe da ESF e o conhecimento aprofundado sobre a temática, fazendo com que oportunidades sejam perdidas, proporcionando uma baixa adesão vacinal (Dominguez et al., 2017).

O estudo 6 apontou que muitas equipes operam com deficiência, tanto estruturais quanto aquelas ligadas às atividades desenvolvidas pelos profissionais. A falta de orientação dada aos pais durante as visitas à sala de vacina detectada nesse estudo é uma constatação inaceitável em serviços que primam pela promoção da saúde, que têm como alicerce a educação em saúde. Atividades como visita domiciliar são decisivas na conquista do vínculo entre profissionais e famílias (Fernandes et al., 2015).

Com relação ao acompanhamento da saúde da criança, o aspecto que requer maior atenção por contribuir para o atraso vacinal, foi o número de consultas de puericultura. Tanto profissionais quanto pais/responsáveis focam o cuidado em situações de agravo à saúde da criança, negligenciando a atenção primária da saúde e o acompanhamento periódico. Os profissionais atribuem esse fator à sobrecarga de trabalho, ao despreparo e à escassez de recursos, a fim de justificar falhas na verificação das cadernetas das crianças durante as consultas (Fernandes et al., 2015).

No estudo 7 ratifica-se os demais estudos: para se obter maior êxito na cobertura vacinal, cabe aos profissionais de saúde uma participação mais ativa na busca de crianças em falta com a vacinação, por meio da revisão sistemática dos cartões, de uma maior efetividade nas visitas domiciliares, cabendo um maior comprometimento aos agentes de saúde. Outra estratégia para o aumento da cobertura vacinal é a melhoria das atividades de educação em saúde, com o intuito de difundir informações aos grupos-alvos, uma vez que a falta de conhecimento e as crenças da população contribuem para o atraso vacinal (Cardoso $e t$ al., 2015).

\section{Conclusão}

Verificou-se que os estudos elucidaram que a imunização é de suma importância para a saúde pública e que a cobertura vacinal é um indicador de qualidade sobre a completude do Calendário Nacional de Vacinação, respeitando a faixa etária, intervalo entre as doses e as oportunidades vacinais. Os autores revelam também que a redução da cobertura vacinal se tem por causas multifatoriais.

Aponta-se que a falta de orientação dos pais/responsáveis sobre a relevância da imunização, famílias numerosas, com baixa escolaridade e renda per capita diminuída atrelados à descrença na veracidade da vacinação ou a recusa por receio dos efeitos adversos evidenciou-se a tendência ao atraso vacinal. Destaca-se a importância de inserir esses responsáveis frente à imunização dessas crianças, enfatizando a necessidade do fortalecimento do vínculo entre os responsáveis/pais e profissionais de saúde, fazendo com que se sintam acolhidos na ESF, evitando-se, assim, a baixa adesão a cobertura vacinal.

Por outro lado, tem os fatores estruturais, que requerem mais atenção por parte dos administradores, a oferta de ESF com falhas estruturais, sendo muitas vezes instaladas em locais provisórios sem uma adequada logística, o que inviabiliza o acesso da clientela. Aa falta de insumos e armazenamento inadequado dos imunobiológicos também fazem com oportunidades sejam perdidas. Assim, vê-se a necessidade de facilitar o acesso e aperfeiçoando das estruturas físicas e do Sistema Nacional de Informação sobre Imunização, tornando-o mais consistente e acessível a fim de obter coberturas cada vez mais acuradas em nível nacional.

O estudo evidenciou também a inaptidão por parte dos profissionais de saúde, onde os mesmos atribuem esse fator à sobrecarga de trabalho, ao despreparo e à escassez de recursos a fim de justificar falhas na verificação das cadernetas das crianças durante as consultas e até mesmo o manuseio e armazenamento dos imunobiológicos. Salienta-se a necessidade de proporcionar uma formação acadêmica com maior embasamento e incentivar a educação continuada dos profissionais, evitando assim, diagnósticos de doenças e falsas contraindicações. 
A prática de vacinar implica em uma convivência entre os responsáveis pela criança e os profissionais de saúde. Para que essa relação seja harmoniosa faz-se necessário a busca ativa de crianças com atraso vacinal por parte dos profissionais de saúde, a visita domiciliar e o incentivo a participação das consultas de puericultura, que são exemplos de métodos que podem ser aprimorados. Outro mecanismo que pode ser empregado é a melhoria das atividades de educação em saúde, com a finalidade de disseminar informações aos grupos-alvos.

Compreende-se que, apesar dos importantes achados desse estudo que subsidiarão a gestão e políticas voltadas à CV, há algumas limitações, que não viabilizam o estudo. Uma limitação é a escassez de estudos realizados nos últimos 5 anos que poderiam apresentar mudanças significativas no contexto atual. Diante do exposto, evidenciando a relevância e o impacto que a imunização exerce sobre a saúde, é indispensável que informações concisas e atuais sobre imunização sejam compartilhadas, assim como o desenvolvimento de estudos que possam colaborar com melhorias e a elucidação de outros desafios relacionados às ações de imunização.

\section{Referências}

Brasil (2015). Verificação da Situação Vacinal. Versão Preliminar. Programa Saúde na Escola. Caderno Temático. http://189.28.128.100/dab/docs/portaldab/documentos/cadernotematicoverificacaosituacaovacinal.pdf

Brasil (2007). Inquérito de Cobertura Vacinal nas áreas urbanas das capitais Brasil. Cobertura Vacinal. https://bvsms.saude.gov.br/bvs/publicacoes/inquerito_cobertura_vacinal_urbanas.pdf

Brasil (2003). Programa Nacional de Imunizações: 30 anos. Ministério da Saúde. http://pni.datasus.gov.br/apresentação.asp

Cardoso, M. D. T. et al (2015). Avaliação da Cobertura Vacinal em Crianças de 2 Meses a 5 anos na Estratégia Saúde da Família. Rev. APS. 18(3). https://periodicos.ufjf.br/index.php/aps/article/view/15346

Comitê consultivo nacional para vacinas (2013). Protegendo a saúde pública: funções críticas do Programa de Imunização da Seção 317 - um relatório do Comitê Consultivo Nacional de Vacinas. Rev. Saúde Pública, 128(2), 78-95. https://www,ncbi.nlm.nih.gov/pmc/articles/PMC3560865.

Dominguez, G. C. S. et al (2017). Retraso del esquema vacunal en niños menores de 5 años en zona marginal. Rev. Nac. 9(1), 35-48. http://scielo.iics.una.py/scielo.php?script=sci_arttext\&pid=S2072-81742017000100035\&lng=en\&nrm=iso

Feijó, R. B. \& Sáfadi, M. A. P. (2006). Imunizações: três séculos de uma história de sucessos e constantes desafios. J. Pediatr. 82(3, supl. P. s1-s3). http://www.sciele.br/scielo.php?script=sci_arttext\&pid=S0021-75572006000400001\&Ing=en\&nrm=iso

Feitosa, L.G. et al (2019). Immunization against human papilomavirus in municipal schools. Journal of Nursing UFPE. 13(set.). ISSN 1981-8963. https://periodicos.ufpe.br/revistas/revistaenfermagem/article/view/241812/33384

Fernandes, A. C. N. et al (2015). Análise da situação vacinal de crianças pré-escolares em Teresina (PI). Revista Brasileira de Epidemiologia. 18(4), 870-882. https://doi.org/10.1590/1980-5497201500040015

Ferreira, V. L. R. et al (2018). Avaliação de coberturas vacinais de crianças em uma cidade de médio porte (Brasil) utilizando registro informatizado de imunização. Cad. Saúde Pública. 34(9). http://www.scielo.br/scielo.php?script=sci_arttext\&pid=S0102-311X2018000905002\&lng=pt\&nrm=iso. Epub 06, Set.

Lemos, D. (2011). Você já se vacinou? iSaúde. https://www.isaude.com.br/noticias/detalhe/noticia/voce-ja-se-vacinou/

Mota, M. F. F. (2016). Situação vacinal e os fatores associados à não vacinação das crianças menores de 24 meses residentes em Fortaleza. 150 f.: il. http://www.repositorio.ufc.br/handle/riufc/57403

Oliveira, V. G. et al (2010). Vacinação: o fazer da enfermagem e o saber das mães e/ou cuidadores. Rev. Rene, v. 11, 133-141; número especial. https://repositorio.ufrn.br/jspui/bitstream/123456789/18279/1/AkemiMA_Vacina\%c3\%a7\%c3\%a3o\%20o\%20fazer\%20da\%20enfermagem\%20e\%20o\%20sab er\%20das\%20m\%c3a3es\%20eou\%20cuidadores_478-1823-1-PB.pdf

Opas - Organização Pan-Americana da Saúde (2020). Pandemia de COVID-19 leva a grande retrocesso na vacinação infantil, mostra novos dados da OMS e UNICEF. https://www.paho.org/pt/noticias/15-7-2021-pandemia-covid-19-leva-grande-retrocesso-na-vacinacao-infantil-mostram-novos.

Pompeo, D. A., Rossi, L. A. \& Galvão, C. M. (2009). Revisão integrativa: etapa inicial do processo de validação de diagnóstico de enfermagem. Acta paul. Enferm. 22(4). http://www.scielo.br/pdf/ape/v22n4/a14v22n4.pdf

Rocha, G. (2015). Programa Nacional de Imunizações (PNI). Blog da Saúde. http://www.blog.saude.gov.br/index.php/entenda-o-sus/50027-programa-nacionalde-imunizacoes-pni

Sato, A. P. S. Qual a importância da hesitação vacinal na queda das coberturas vacinais no Brasil? Rev Saude Publica. 2018; 52-96. https://www.scielosp.org/pdf/rsp/2018.v52/96/pt

Silveira, A. S. A. et al (2007). Controle de vacinação de crianças matriculadas em escolas municipais da cidade de São Paulo. Rev. esc. enferm. 41(2), 299-305. http://www.scielo.br/scielo.php?script=sci_arttext\&pid=S0080-62342007000200018\&Ing=pt\&nrm=iso. 
Research, Society and Development, v. 11, n. 2, e49611225455, 2022

(CC BY 4.0) | ISSN 2525-3409 | DOI: http://dx.doi.org/10.33448/rsd-v11i2.25455

Tauil, M. C. et al (2017). Coberturas vacinais por doses recebidas e oportunas com base em um registro informatizado de imunização, Araraquara-SP, Brasil, 2012-2014. Epidemiol. Serv. Saúde. 26(4), 835-846. http://www.scielo.br/scielo.php?script=sci_arttext\&pid=S2237-96222017000400835\&lng=en\&nrm=iso

Texeira, A. M. S., \& Rocha, C. M. V. (2010). Vigilância das coberturas de vacinação: uma metodologia para detecção e intervenção em situações de risco. Epidemiol. Serv. Saúde. 19(3), 217-226. https://bvsms.saude.gov.br/bvs/publicacoes/vigilancia_coberturas_vacinacao.pdf 